\title{
Strategy for the simulation and analysis of longitudinal phenotypic and genomic data in the context of a temperature $x$ humidity-dependent covariate
}

\author{
T. Yin, ${ }^{\star}$ E. C. G. Pimentel, ${ }^{\star}$ U. König v. Borstel,† and S. König ${ }^{\star 1}$ \\ *Department of Animal Breeding, University of Kassel, 37213 Witzenhausen, Germany \\ †Department of Animal Breeding, Göttingen University, 37075 Göttingen, Germany
}

\begin{abstract}
A simulation study was conducted to evaluate the performance of genomic random regression models for the continuous environmental descriptor temperaturehumidity index (THI). Statistically innovative aspects of the study included the combined simulation of both longitudinal phenotypic data representing the same trait in the course of THI and genomic data. The longitudinal trait was simulated (phenotypically expressed) at 5 different values of THI. For a moderate heritability trait, heritabilities were $0.30,0.35,0.40,0.40$, and 0.35 for THI of $15,30,45,60$ and 75 , respectively. In a consecutive run, low heritabilities of $0.05,0.1$, $0.15,0.15$, and 0.10 were simulated, respectively. On the genomic level, simulation combined high and low linkage disequilibrium with 5,000-, 15,000-, and 50,000SNP chip applications to simulate different scenarios of genomic architecture. With regard to data analyses, 2 strategies were applied to evaluate the accuracy of genomic predictions across THI, with special focus on the extreme ends of the environmental scale. In the first strategy, $100,80,50$, or $20 \%$ of phenotypes at THI 75 were deleted randomly and the remaining data set was used to predict the breeding value at THI 75 for non-phenotyped, but genotyped cows. In the second strategy, 1,600 cows had complete information (genotypes and phenotypes) and 400 cows were genotyped, but with missing phenotypes for all THI. For the first strategy and without phenotypic observations at THI 75 , accuracies of genomic predictions were lower than 0.34 . When only $20 \%$ of cows had phenotypic records at THI 75 , accuracies increased $(\sim 0.60)$. Such a small proportion of phenotyped cows was sufficient to predict reliable genomic breeding values for cows without phenotypes for extreme THI. For the second strategy, also for low linkage disequilibrium combined with a low density 5,000-SNP chip, the average accuracy of
\end{abstract}

Received June 14, 2013.

Accepted December 12, 2013

${ }^{1}$ Corresponding author: sven.koenig@uni-kassel.de genomic predictions was 0.52 , which is substantially higher than accuracies based on pedigree relationships. From a practical perspective, genomic random regression models can be used to predict genomic breeding values for scarce phenotypes (e.g., novel traits) traits measured in extreme environments, or traits measured late in life, such as longevity.

Key words: genomic selection, genotype by environment interaction, random regression

\section{INTRODUCTION}

Methods for dealing with longitudinal data in genetic evaluations have evolved from the use of repeatability models with permanent environmental effects or multiple-trait models with covariance matrices (Henderson, 1984) to random regression models (RRM; Schaeffer and Dekkers, 1994) with covariance functions (Kirkpatrick et al. 1990). The use of RRM for analyzing longitudinal production data are a standard in genetic evaluations for dairy cattle worldwide, because such models provide an overview of genetic parameters and breeding values across the whole lactation trajectory. Additionally, further applications of RRM to describe performances over a range of environments in reaction norm studies have been proposed. Such models are interesting with regard to genotype by environment interactions, where different environments can be defined on a continuous scale. Ravagnolo and Misztal (2000) estimated variance components for milk production traits at different levels of heat stress, defined by a temperature-humidity index (THI). In more recent studies, RRM were further elaborated by defining THI as an environmental covariate (Aguilar et al., 2009; Brügemann et al., 2011). The basic idea of RRM applications is to depict the physiological background or genetic mechanisms of traits in a quantitative genetic context, meaning that different genes are switched on or off with, for example, aging of the animal or with environmental changes. Substantial changes of both quantitative genetic parameters and gene expression profiles by inducing heat stress were shown in fertility 
traits of mice (Cammack et al., 2006, 2009). Genetic studies on heat stress in dairy cattle have an important practical background with regard to semen and livestock exports. For example, the German dairy cattle industry, and especially the dairy cattle breeding organization Masterrind $\mathrm{GmbH}$ (Verden, Germany), is strongly involved in exports of livestock and sire semen. Target countries include tropical countries located in Asia, Africa, and South America. In 2012, a total of 26,249 heifers were exported to these countries (DHV, 2013). The tropical and hot climates in the importing countries causes heat stress in the cows, especially when the THI rises above 72 (Bohmanova et al., 2005). In contrast, performance testing within Germany exhibits a shift from population-wide recording schemes toward so-called selected "contract herds" (Schierenbeck et al., 2011). Contract herds are characterized by superior feeding, management, and housing conditions, and by substantially lower THI levels realized by, for example, housing systems with integrated cooling techniques (Brügemann et al., 2012).

Classically, RRM applications (also when studying genotype by environment interactions) are based on longitudinal phenotypic records combined with genetic relationships from pedigree data. Nowadays, the availability of high-throughput genotyping technologies with decreasing costs encourages dairy cattle farmers worldwide to genotype an increasing percentage of cows, heifers, and female calves. Especially for novel traits, reliable, conventional EBV of bulls do not exist. Hence, basing genomic selection on calibration groups of cows might be a promising alternative (Buch et al., 2012). Examples include health traits (Pintus et al., 2013), and traits reflecting energy balance (Verbyla et al., 2010). Furthermore, Misztal et al. (2010) suggested the inclusion of genomic information to improve the accuracy of genetic evaluations of young animals for heat tolerance. Availability of cow genotypes combined with longitudinal phenotypic data enable the application of genomic RRM (gRRM) to estimate genomic breeding values $(\mathbf{G B V})$ for scarcely recorded traits, or for environmental descriptors that are not or poorly represented in a data set. In this latter context, Suchocki and Szyda (2011) estimated SNP effects over time by applying a mixed model with orthogonal polynomials and genotyped animals for longitudinal growth data. An alternative might be the direct estimation of GBV using gRRM and BLUP. Simulations are a powerful tool to evaluate a broad variety of statistical procedures based on longitudinal phenotypic and genomic data and, in consequence, to study the effects of various scenarios on selection and mating schemes. Accuracies of genomic predictions strongly depend on technical parameters (e.g., size of calibration group and pattern of SNP chips), the quantity and quality of phenotypic data, quantitative genetic parameter estimates, and the genomic architecture of the trait. To our knowledge, no simulation package exists that simultaneously addresses those aspects for longitudinal data structures and directly provides true breeding values (TBV), GBV, and phenotypes in the course of a continuous environmental descriptor.

Consequently, the objectives of the present study were to (1) develop a framework for the simulation of longitudinal phenotypic data combined with highthroughput genotypes, (2) evaluate the performance of a gRRM in the context of reaction norms, and (3) investigate the accuracy of genomic predictions for cows that are poorly or not at all represented in the group of cows with records for environmental descriptors. For illustration and based on experiences from previous studies, the environmental descriptor THI was chosen, but applications to further problems will be discussed. The study was performed by varying the assumptions related to the genomic architecture of traits.

\section{MATERIALS AND METHODS}

\section{Simulation of Populations}

Populations were simulated using the software QMSim (Sargolzaei and Schenkel, 2009). With QMSim, the simulation process is divided in 2 stages. First, a historical population is simulated for several generations to generate a desired level of linkage disequilibrium (LD). In a second step, using animals from the last historical generation as founders, further recent populations are simulated for a desired number of generations. Within this second simulation step, population parameters can be varied to generate the appropriate population structure. In the present study, 2 different types of historical populations were simulated to create scenarios with either low or high LD. For both low-LD and high-LD scenarios, 10 recent generations were simulated based on the parameters as specified in Table 1.

Additionally, Table 1 summarizes the parameters of the simulated genome. The simulated genome consisted of 30 chromosomes of $100 \mathrm{cM}$ each. The number of QTL per chromosome was set to 10 and QTL positions on the chromosome were randomly assigned. Effects of QTL alleles were drawn from a gamma distribution with a shape parameter 0.4. The number of QTL alleles at each locus was randomly assigned and was 2,3 , or 4. To achieve resemblance with different densities of SNP chips, 3 scenarios with respect to the number of markers on the genome were simulated. The simulation of 167,500 , and 1,667 biallelic markers per chromosome depicts applications with 5,000 (5K)-, 15,000 (15K)-, 
Table 1. Parameters of the simulation process

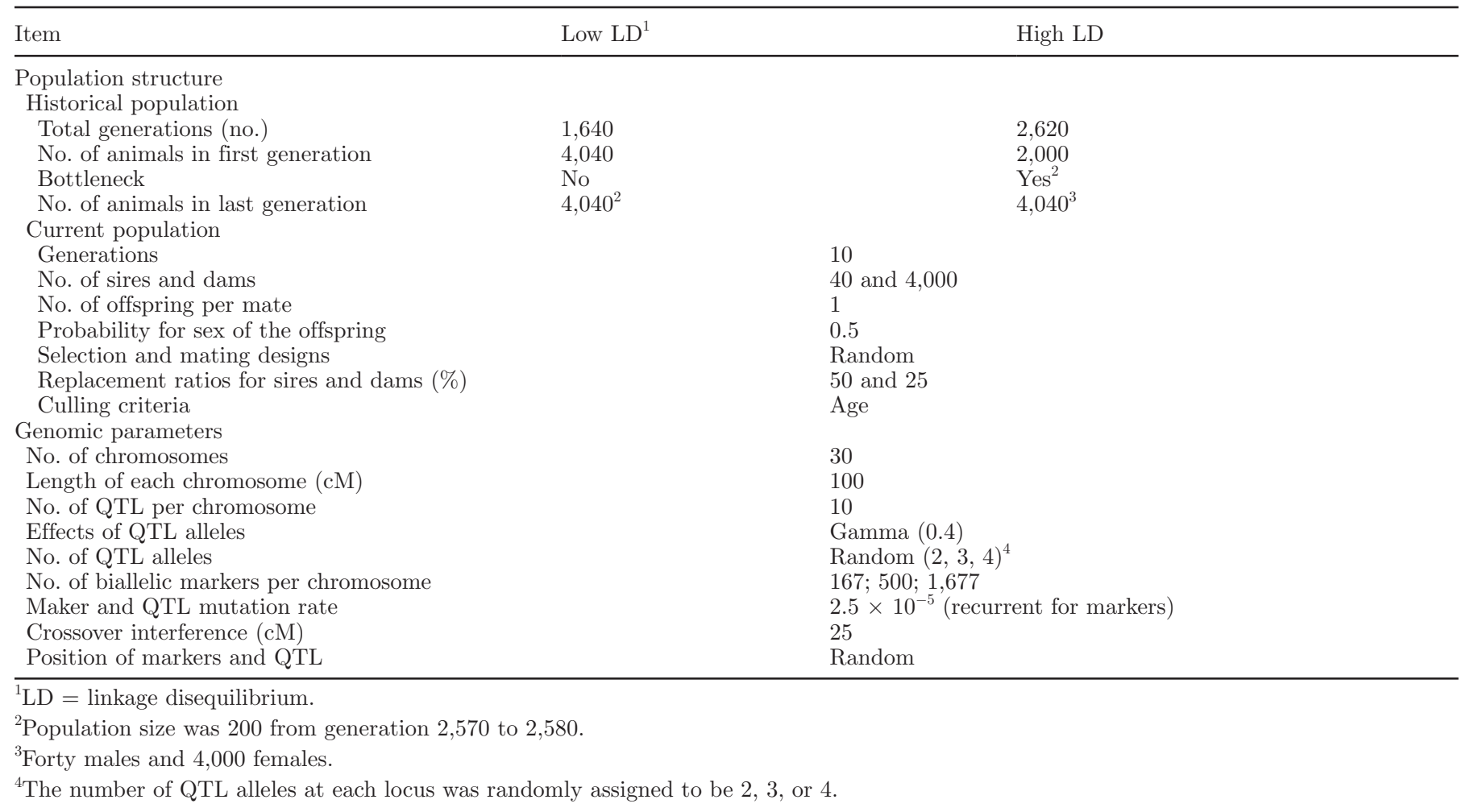

and 50,000 (50K)-SNP chips, respectively. Both marker and QTL mutation rates were $2.5 \times 10^{-5}$, whereas recurrent mutation was only allowed for markers. The crossing-over interference was defined as $25 \mathrm{cM}$ (i.e., a random placement of crossing over along chromosomes can be interrupted if the distance between a pair of a crossing over is smaller than $25 \mathrm{cM}$ ).

\section{Simulation of Longitudinal Data}

The QMSim output, which included QTL genotypes, QTL effects, and SNP markers, was modified by our own programming to produce longitudinal data records for 2,000 cows in the last generation. In total, $300 \mathrm{QTL}$ controlling the trait of interest (i.e., heat tolerance per se or performances at different heat stress levels) were randomly divided into 10 groups, with an even group size of 30 QTL. True breeding values of cows in 10 different groups were based on the 30 different QTL in each group and were calculated using the corresponding QTL effects as produced from the QMSim program. Following the protocol of Brügemann et al. (2011), the environmental continuous descriptor THI varied from 15 to 75 in increments of 15 . To model changes in gene expression in the course of THI, only QTL in groups 1 to 6 were assigned to express at THI 15, indicating that TBV of 2,000 cows at THI 15 (TBV_THI15) were the sum of effects for each QTL in groups 1 to 6. At THI 30, only QTL in groups 2 to 7 contributed to TBV_THI30, and so on (Table 2). This simulation strategy resulted in 5 groups of overlapping QTL (i.e., 150 QTL) between adjacent THI levels. For example, QTL in groups 2 to 6 expressed simultaneously at THI 15 and THI 30. Even the 2 most extreme THI levels (THI 15 and THI 75) had 2 groups of QTL in common (groups 5 and 6). Simulation was done in this way to smooth the correlations across the THI levels. Furthermore, this simulation strategy reflects the fact that the trait has some basic genetic background effecting its phenotypic expression also with environmental changes. Consequently, the true genetic correlations between THI levels were determined by the number of overlapping QTL groups, which depicts the basic idea and flexibility of our simulation strategy.

Phenotypes were created by adding a residual to TBV at THI 15 to 75 according to a set of defined heritabilities. Heritabilities for the 5 chosen THI (15, $30,45,60$, and 75$)$ were $0.30,0.35,0.40,0.40$, and 0.35 , respectively, for a moderate heritability trait, and 0.05 , $0.10,0.15,0.15$, and 0.10 , respectively, in a consecutive run for a low heritability trait. In total, the number of phenotypic records for the 2,000 cows was 10,000, indicating that each cow had 5 repeated measurements (i.e., the same trait expressed at 5 different THI). Lon- 
Table 2. Expression of groups of QTL at different temperature-humidity index (THI) levels for mimicking the physiological background of gene expression

\begin{tabular}{lllllllllll}
\hline & \multicolumn{10}{c}{ QTL group } \\
\cline { 2 - 10 } THI & 1 & 2 & 3 & 4 & 5 & 6 & 7 & 8 & 9 & 10 \\
\hline 15 & $\mathrm{X}^{1}$ & $\mathrm{X}$ & $\mathrm{X}$ & $\mathrm{X}$ & $\mathrm{X}$ & $\mathrm{X}$ & & & & \\
30 & & $\mathrm{X}$ & $\mathrm{X}$ & $\mathrm{X}$ & $\mathrm{X}$ & $\mathrm{X}$ & $\mathrm{X}$ & & & \\
45 & & $\mathrm{X}$ & $\mathrm{X}$ & $\mathrm{X}$ & $\mathrm{X}$ & $\mathrm{X}$ & $\mathrm{X}$ & & \\
60 & & & $\mathrm{X}$ & $\mathrm{X}$ & $\mathrm{X}$ & $\mathrm{X}$ & $\mathrm{X}$ & $\mathrm{X}$ & $\mathrm{X}$ \\
75 & & & & $\mathrm{X}$ & $\mathrm{X}$ & $\mathrm{X}$ & $\mathrm{X}$ & $\mathrm{X}$ & $\mathrm{X}$ \\
${ }^{1} \mathrm{X}=$ QTL expressed in the group. & & & & & & & & &
\end{tabular}

gitudinal data records are characterized by phenotypic and genetic relationships along the trajectory of the continuous environmental descriptor THI. Genetic and phenotypic correlations between 5 different THI averaged across 10 replicates are listed in Table 3 for the moderate-heritability scenario and in Table 4 for the low-heritability scenario. Higher genetic correlations for protein yield between adjacent THI than for very different THI were also found by Brügemann et al. (2011) and reflect the physiological and practical background of our simulation.

\section{Statistical Model}

A gRRM was applied to analyze the simulated longitudinal data using the AI-REML algorithm and applying the DMU package (Madsen and Jensen, 2010). In matrix notation, the statistical model was

$$
\mathbf{y}=\mathbf{X b}+\mathbf{Z}_{1} \mathbf{a}+\mathbf{Z}_{2} \mathbf{p}+\mathbf{e}
$$

where $\mathbf{y}=$ vector of phenotypic observations for cows, $\mathbf{b}=$ vector of fixed regressions on THI using thirdorder Legendre polynomials, $\mathbf{a}=$ vector of additive genetic effects for random regression coefficients on THI using third-order Legendre polynomials, $\mathbf{p}=$ vector of permanent environmental effects, $\mathbf{e}=$ vector of random residual effects, and $\mathbf{X}, \mathbf{Z}_{1}$, and $\mathbf{Z}_{2}=$ incidence matrices for $\mathbf{b}, \mathbf{a}$, and $\mathbf{p}$, respectively. The genetic relationship matrix between individuals was either built

Table 3. Heritabilities (diagonal), genetic (above diagonal), and phenotypic (below diagonal) correlations of the simulated longitudinal data between different temperature-humidity indices (THI ${ }^{1}$

\begin{tabular}{lccccc}
\hline & \multicolumn{5}{c}{ THI } \\
\cline { 2 - 6 } THI & 15 & 30 & 45 & 60 & 75 \\
\hline 15 & 0.30 & 0.86 & 0.69 & 0.54 & 0.38 \\
30 & 0.28 & 0.35 & 0.83 & 0.68 & 0.53 \\
45 & 0.24 & 0.32 & 0.40 & 0.84 & 0.69 \\
60 & 0.19 & 0.25 & 0.35 & 0.40 & 0.85 \\
75 & 0.13 & 0.18 & 0.27 & 0.32 & 0.35 \\
\hline
\end{tabular}

${ }^{1}$ Simulation of a moderate-heritability trait. via pedigrees including 10 generations (A) or via SNP data of cows $(\mathbf{G})$. In the following, scenarios based on genomic data are labeled $\mathrm{G}_{-} *$, where the asterisk (*) specifies the level of LD and the size of the SNP chip. The pedigree-based scenario is labeled $\mathbf{A}$. The $\mathbf{G}$ matrix was computed from the Gmatrix program $(\mathrm{Su}$ and Madsen, 2011) based on the method proposed by VanRaden (2008). Markers with minor allele frequency lower than 0.05 were deleted. A small value (0.01) was added to the diagonal of the $\mathbf{G}$ matrix to ensure that the matrix was positive definite.

For all scenarios, results from the gRRM were compared by applying a relatively simple genomic repeatability model (gRM). In matrix notation, the gRM was

$$
\mathbf{y}=\mathbf{X} \mathbf{b}+\mathbf{Z}_{1} \mathbf{a}+\mathbf{Z}_{2} \mathbf{p}+\mathbf{e},
$$

where $\mathbf{b}=$ vector of fixed effects of THI levels and $\mathbf{a}$ $=$ vector of additive genetic effects. Other effects were identical to those of the gRRM.

\section{Accuracy of Genomic Predictions}

Conventional EBV and GBV in the course of the environmental descriptor were calculated by multiplying random regression coefficients for additive genetic effects for each cow with corresponding Legendre polynomials for THI 15, 30, 45, 60, and 75. Accuracies of estimates were correlations between TBV and GBV (or EBV). For calculating the accuracy of genomic predic-

Table 4. Heritabilities (diagonal), genetic (above diagonal), and phenotypic (below diagonal) correlations of the simulated longitudinal data between different temperature-humidity indices $(\mathrm{THI})^{1}$

\begin{tabular}{llllll}
\hline & \multicolumn{5}{c}{ THI } \\
\cline { 2 - 6 } THI & 15 & 30 & 45 & 60 & 75 \\
\hline 15 & 0.05 & 0.86 & 0.69 & 0.54 & 0.38 \\
30 & 0.05 & 0.10 & 0.83 & 0.68 & 0.53 \\
45 & 0.06 & 0.10 & 0.15 & 0.84 & 0.69 \\
60 & 0.05 & 0.08 & 0.13 & 0.15 & 0.85 \\
75 & 0.01 & 0.05 & 0.08 & 0.10 & 0.10 \\
\hline
\end{tabular}

${ }^{1}$ Simulation of a low-heritability trait. 
tions for cows without phenotypes, 2 strategies of data analysis were applied. In the first strategy, 100, 80, 50, or $20 \%$ of records only at THI 75 were deleted randomly and the remaining data set was used to predict GBV (or EBV) for non-phenotyped cows at THI 75. In a second strategy, complete information (genotypes and phenotypes) was available from 1,600 cows (training set), and phenotypes were missing from 400 cows (validation set) for all THI. Within this second strategy, 2 approaches were simulated to allocate cows to the 2 sets. In the first approach, 1,600 cows were randomly selected as reference animals and used to predict GBV (or EBV) for the 400 cows without phenotypes. An alternative approach to allocate cows in training and validation sets was accomplished by mimicking low relationships between candidates in both groups. The alternative approach implied that the training set only included daughters of 8 sires (400 cows), and daughters from the other 32 sires (1,600 cows) were represented in the validation set. The 2 different strategies and the 2 approaches within the second strategy for allocating cows in training and validation sets are illustrated in Figure 1.

\section{RESULTS AND DISCUSSION}

\section{Genomic Architecture}

Degree of LD between all possible SNP pairs was measured by using the squared correlation coefficient $\left(\mathbf{r}^{2}\right.$; Hill and Robertson, 1968). In total, 2,000 cows with genomic information were used to compute $r^{2}$.
The bottleneck in the historical population was a crucial factor influencing LD between markers. Linkage disequilibrium strongly determines the genomic architecture of traits, which has a substantial effect on (1) the choice of the method for genomic evaluations and (2) the reliability of its outcome (e.g., Daetwyler et al., 2010). The average $r^{2}$ from 1 of the 30 chromosomes across 10 replicates between SNP pairs for both the low LD and high LD scenarios combined with 5K-, 15K-, and 50K-SNP chip applications are plotted against a map distance of up to $2 \mathrm{Mb}$ (Figure 2). Generally, average $\mathrm{r}^{2}$ for both high- and low-LD scenarios decreased with an increase in map distances between SNP. We observed substantial declines with regard to average $\mathrm{r}^{2}$ for distances in the range from 0 to $0.8 \mathrm{Mb}$, but the declines were low to moderate for distances ranging between 0.8 and $2.0 \mathrm{Mb}$. Such an exponential decrease for $\mathrm{r}^{2}$ was shown in several studies (e.g., Jiménez-Montero et al., 2013). The average $\mathrm{r}^{2}$ for high-LD scenarios were larger than for low-LD scenarios, especially for a small distance between 2 SNP. However, if the distance between markers was larger than $5.0 \mathrm{Mb}$, no difference in $\mathrm{r}^{2}$ was found between high-LD and low-LD scenarios. The average $r^{2}$ was very close to zero $(0.005)$ when distances were $100 \mathrm{Mb}$.

\section{Accuracies of Breeding Values for Cows With Phenotypes}

Accuracies of GBV of cows with phenotypes at all THI levels were calculated for both scenarios (high and

Entire simulated population with 2,000 genotyped cows with 5 repeated
measurements per cow
No. of the

Figure 1. Strategies and approaches within strategies for defining subsets for genomic prediction with genomic random regression models (cows given in the dashed-line box and in the gray box indicate the animals without phenotypes for the 2 different strategies). THI $=$ temperature-humidity index. 


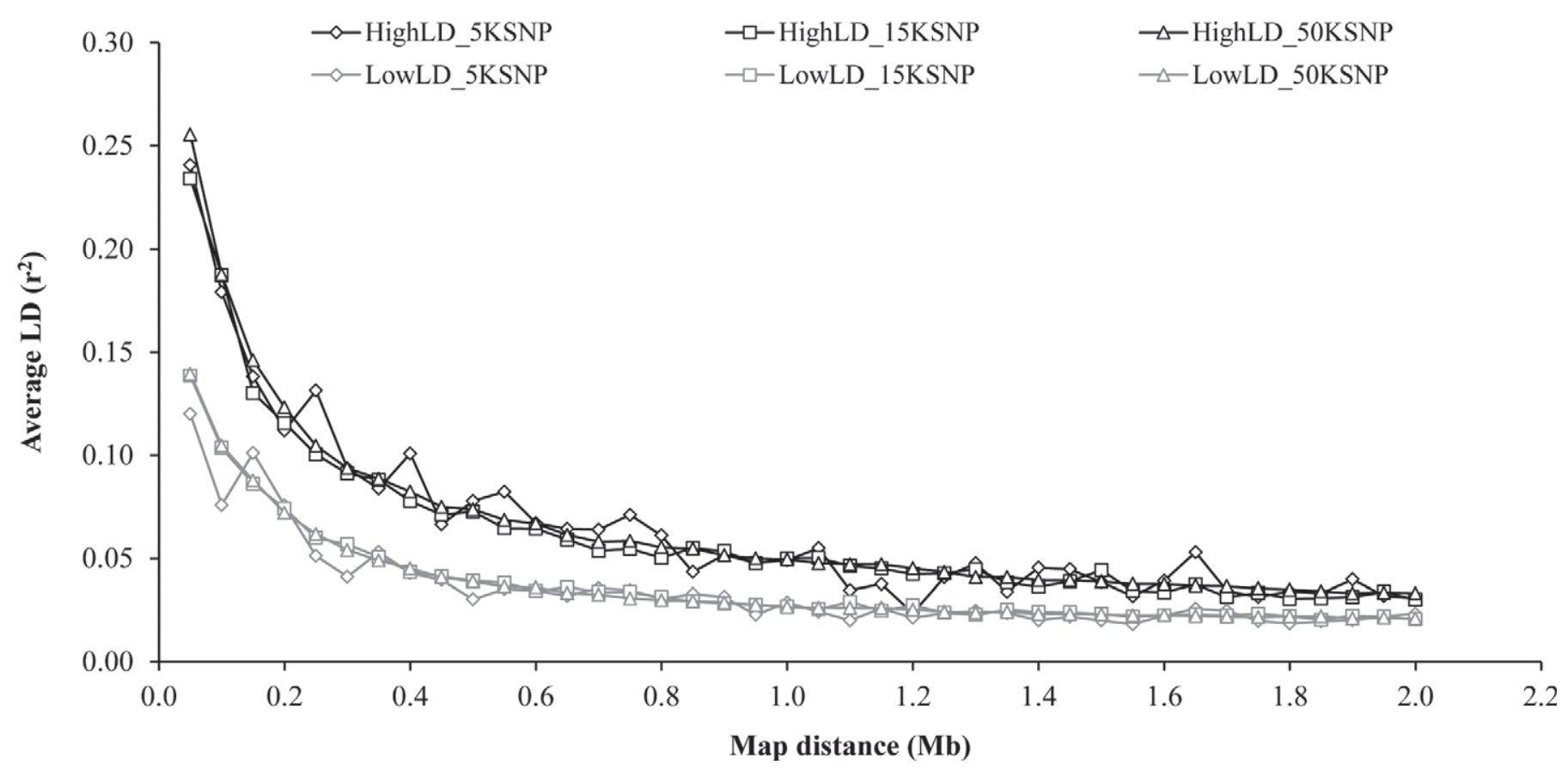

Figure 2. Average linkage disequilibrium [LD; squared correlation coefficient $\left.\left(\mathrm{r}^{2}\right)\right]$ between SNP markers dependent on their map distance for the different sets of simulated marker data. $5 \mathrm{~K}=5,000 ; 15 \mathrm{~K}=15,000 ; 50 \mathrm{~K}=50,000$.

low LD) combined with different sizes of SNP chips (5K, $15 \mathrm{~K}$, and $50 \mathrm{~K})$. Average accuracies from 10 replicates for the longitudinal trait with moderate heritability using the complete data set of 2,000 cows with phenotypes and genotypes are shown in Figure 3. Generally, accuracies of GBV and EBV increased with increasing heritabilities and were highest for a heritability of 0.40 at THI 45. Interestingly, accuracies at THI 30 are marginally higher than at THI 75 even though the same heritability $\left(\mathrm{h}^{2}=0.35\right)$ was assumed. To explain this phenomenon, a trait with a constant heritability $\left(\mathrm{h}^{2}=\right.$ 0.35 ) in the course of THI was simulated and accuracies for the 2 scenarios G_HighLD_15KSNP and A (where $\mathrm{G}=$ scenario based on the genetic relationship matrix using the SNP data of cows and $\mathrm{A}=$ scenario based on the pedigree relationship matrix) were calculated. For both scenarios, and assuming identical heritabilities along the trajectory, accuracies of predictions were highest at THI 45. An explanation might be that phenotypic records at THI 45 were genetically highly correlated with records measured at remaining THI (the lowest genetic correlation was 0.68). In contrast, for GBV (or EBV) estimated at THI 15, only data from THI in close proximity to THI 15 (THI 30 and 45) were highly correlated with data from THI 15 (genetic correlation >0.68). Records at the upper end of the environmental scale (THI 60 and 75) only showed moderate genetic correlations of 0.54 and 0.38 , respectively (Table 3).
Accuracies of GBV for scenarios based on genomic relationship matrices were always higher compared with EBV from the pedigree-based scenario A, except for low LD combined with a low-density SNP chip (G_LowLD_5KSNP; Figure 3). The highest accuracy was realized in scenario G_HighLD_50KSNP with a value of 0.82 at THI 45 and 60 . For genomic scenarios, accuracies of GBV increased with increasing LD and increasing size of the SNP chip. A strong and positive relationship between LD and accuracy of GBV was observed in previous studies (e.g., by Zhong et al., 2009). Due to the fact that more markers and QTL are in LD, more markers capture a higher proportion of genetic variance of the trait (Goddard, 2009). Additionally, higher marker density increased the $\mathrm{r}^{2}$ between adjacent markers and the accuracy of genomic prediction (Meuwissen, 2009). As one example in our study, the average $\mathrm{r}^{2}$ for the scenario G_HighLD_50KSNP was 0.26 , but $\mathrm{r}^{2}$ was only 0.11 for the scenario G_HighLD_5KSNP. The corresponding averaged accuracies of genomic predictions for the 2 scenarios were 0.79 and 0.74 , respectively.

Figure 4 depicts accuracies of predictions for different levels of LD and marker density at 5 THI for the lowheritability trait. As expected, accuracies were lower compared with results for the moderate-heritability trait (Figure 3), but revealing identical tendencies with regard to LD and the size of SNP chips. Especially for the low-heritability trait in the range from THI 15 to 45 (Figure 4), genomic scenarios with high LD achieved 


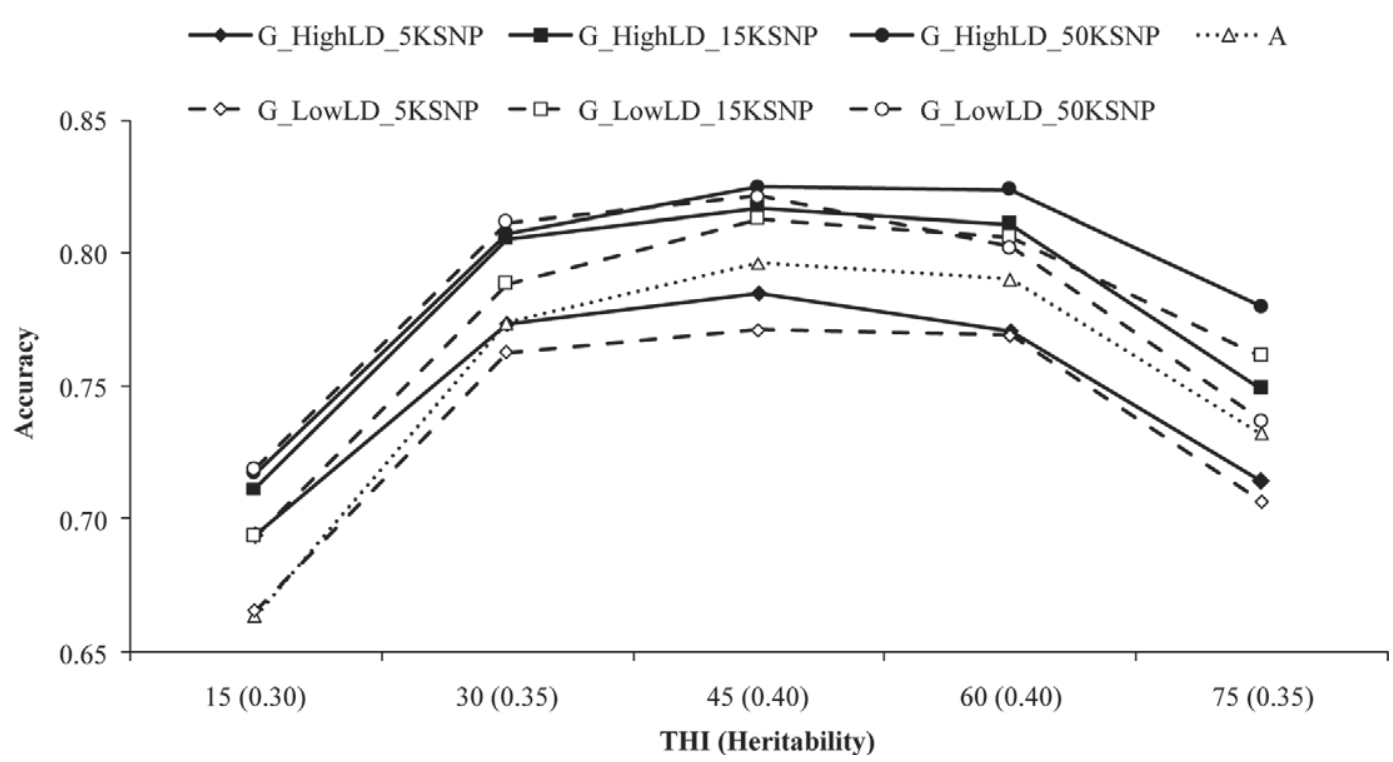

Figure 3. Average accuracies of genomic predictions for a longitudinal trait with heritabilities of $0.30,0.35,0.40,0.40$, and 0.35 for temperature-humidity indices (THI) of $15,30,45,60$, and 75 , respectively. Different scenarios include variation in SNP density [5,000 (5K), 15,000 $(15 \mathrm{~K})$, and $50,000(50 \mathrm{~K})]$ and linkage disequilibrium $(\mathrm{LD}) . \mathrm{G}=$ scenario based on the genetic relationship matrix using the SNP data of cows; $\mathrm{A}=$ scenario based on the pedigree relationship matrix.

substantially higher accuracies of prediction compared with the pedigree-based scenario. Buch et al. (2012) also found that genotyping of cows simultaneously increased accuracies of genomic prediction for traits with low heritability. When basing within-herd replacement decisions of cows on GBV instead on pedigree indices, accuracy of selection and genetic merit of females will be improved with an associated positive effect on farm economy (Weigel et al., 2012).

For the low-heritability trait, the pedigree-based RRM, but especially the more complex gRRM, revealed some convergence problems (i.e., longer computing time was required to meet convergence criteria). Hence, we conclude that more observations are required when applying gRRM to low-heritability traits. Yin et al. (2012) applied RRM to estimate genetic parameters by DIM and by parity for low-heritability functional traits comprising a comparatively small data set of 1,283 cows from low-input systems in Switzerland. In some cases, statistical models not only failed to converge, but also estimates of heritabilities or genetic correlations were extraordinarily high at the extreme ends of the continuous time scale.

\section{Accuracies of Breeding Values for Cows Without Phenotypes}

Figure 5 shows accuracies of breeding values for nonphenotyped cows by altering percentages of cows with phenotypes at an extreme end of the continuous envi- ronmental scale (THI 75). For the moderate-heritability scenario, and without phenotypic records at THI 75, accuracies of predictions at THI 75 were generally low and, on average, at a value of 0.22 (average of all scenarios) with corresponding high standard deviations in the range from 0.19 to 0.33 within scenarios. However, for $20 \%$ of phenotyped cows at THI 75 , accuracies of predictions substantially increased to a moderate value of 0.60 , by decreasing standard deviations (within scenarios, the standard deviation decreased by factor 5 ). An average accuracy of prediction of 0.69 was realized for $80 \%$ of the 2,000 cows with phenotypic records at THI 75 and with the 15K SNP chip used for genotyping. Interestingly, for the scenario without phenotypes at THI 75, accuracies of predictions from the gRM were higher than from the gRRM. For example, for the scenario G_HighLD_15KSNP, accuracies were 0.56 and 0.33 , respectively. In contrast with $20 \%$ or more of phenotyped cows at THI 75, gRRM were superior to gRM. Again, for the scenario G_HighLD_15KSNP, accuracy from the gRRM was 0.63 , and 0.57 from the gRM. Generally, standard deviations of accuracies from the gRM were larger than standard deviations from gRRM applications.

According to the heat stress studies conducted by Brügemann et al. (2012) in Germany, only a limited number of genotyped heifers or cows had phenotypic performances in environments with extremely high THI. Based on the results from our present study, only a small proportion of phenotyped cows (i.e., 20\%) in 


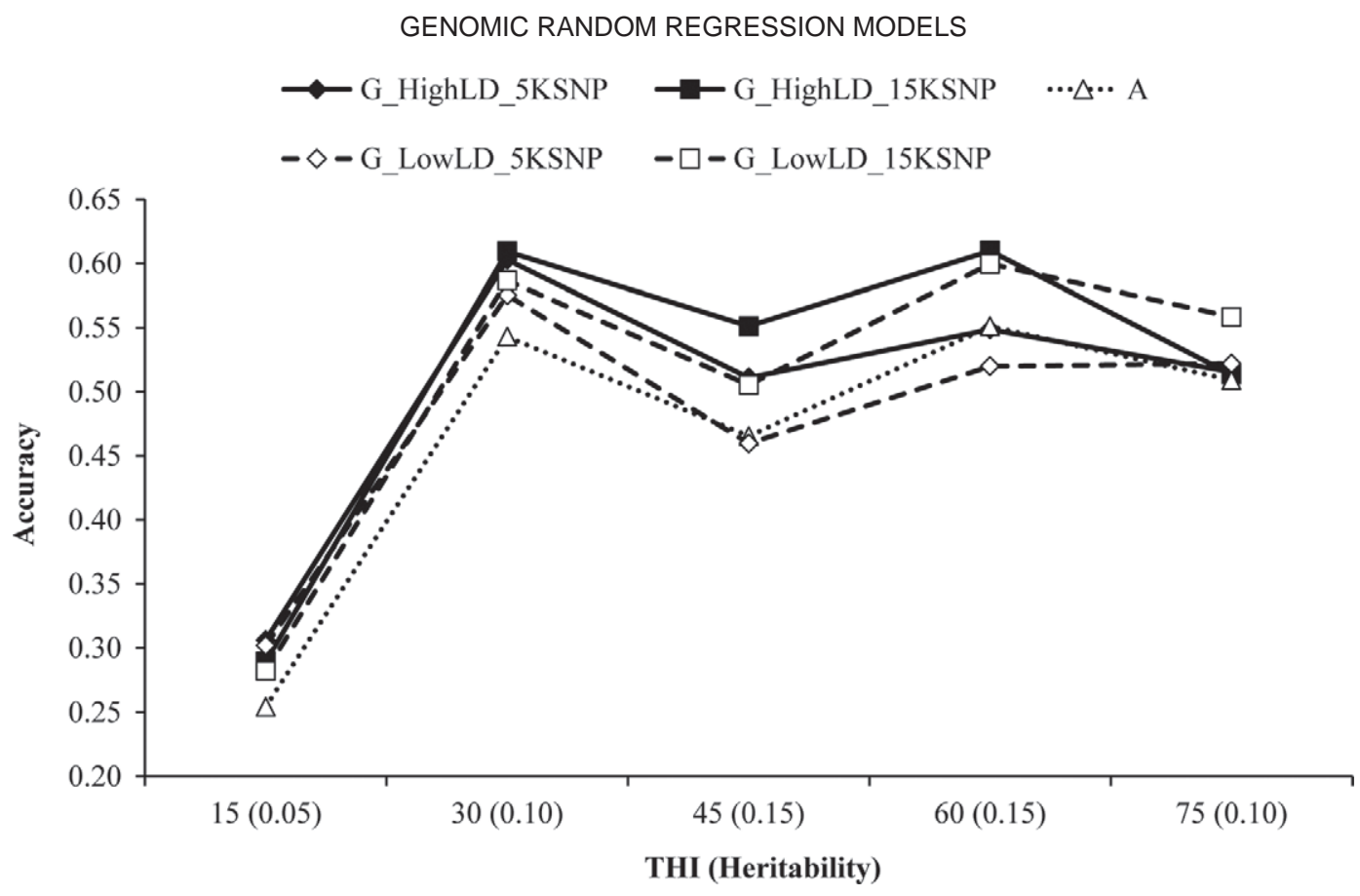

Figure 4. Average accuracies of genomic predictions for a longitudinal trait with heritabilities of $0.05,0.10,0.15,0.15$, and 0.10 for temperature-humidity indices (THI) of $15,30,45,60$, and 75 , respectively. Different scenarios include variation in SNP density [5,000 (5K) and 15,000 $(15 \mathrm{~K})]$ and linkage disequilibrium $(\mathrm{LD}) . \mathrm{G}=$ scenario based on the genetic relationship matrix using the SNP data of cows; A = scenario based on the pedigree relationship matrix.

environments representing heat stress (i.e., THI 75) is required to predict reliable GBV of cows without phenotypes. Without phenotypic records at THI 75 , the maximal accuracy amounts to 0.33 , indicating that RRM or gRRM cannot predict the genetic variance of the trait accurately when there is a complete lack of phenotypes at the extreme ends of a continuous environmental scale. A further practical application of gRRM might be the prediction of GBV for longevity of genotyped heifers or other traits measured late in a dairy cow's life. Such a general application of RRM for predicting longevity was suggested by Schaeffer (2004).

Figure 6 depicts accuracies of predictions for the pedigree-based and for the genomic scenarios with 1,600 cows in a calibration set (i.e., genotypes and phenotypes were available for all THI), and 400 cows in the prediction set (i.e., cows with genotypes but without phenotypes). The scenario high LD using the 15K SNP chip (G_HighLD_15KSNP) provided the highest accuracy of genomic predictions across THI. Average accuracies across replicates and THI were 0.54 for the scenario high LD with the $5 \mathrm{~K}$ SNP chip, 0.58 for the scenario high LD with the 15K SNP chip, 0.49 for scenario A, 0.52 for the scenario low LD with the $5 \mathrm{~K}$ SNP chip, and 0.56 for the scenario low LD with the $15 \mathrm{~K}$ SNP chip. Among the 5 scenarios, again the pedigree-based predictions were less accurate compared with genomic predictions, even compared with low LD in combination with the low-density $5 \mathrm{~K}$ SNP chip (accuracy of genomic prediction ranged between 0.49 and 0.55). Not only gRRM, but also genomic multiple-trait models increase the accuracy of genomic predictions compared with genomic univariate models (Tsuruta et al., 2011), especially when multiple traits are strongly correlated. For an environmental descriptor with only a few environmental levels, also a genomic multiple-trait model can be applied, but not for a continuous environmental descriptor with more levels at closer intervals (as also valid for THI, in practice).

In addition to genetic architecture and marker density, the level of genetic relationships between cows in the defined calibration set and cows in the prediction set is a crucial factor influencing the accuracy of genomic predictions in gRRM. The genotyped 2,000 cows in this study were progeny from 40 sires, with an average of 50 daughters per sire. For the first approach of the second strategy (Figure 1), each sire had, on average, 10 daughters in the calibration set and 40 daughters in the training set. Therefore, cows in the prediction set were genetically related to their half-sibs in the prediction set and these close relationships might have increased the accuracy of genomic prediction. To verify accuracies caused by LD and marker density only, a large genetic distance between both groups should be achieved (Bolormaa et al., 2010). This distance was maximized by deleting phenotypes of daughters from 8 


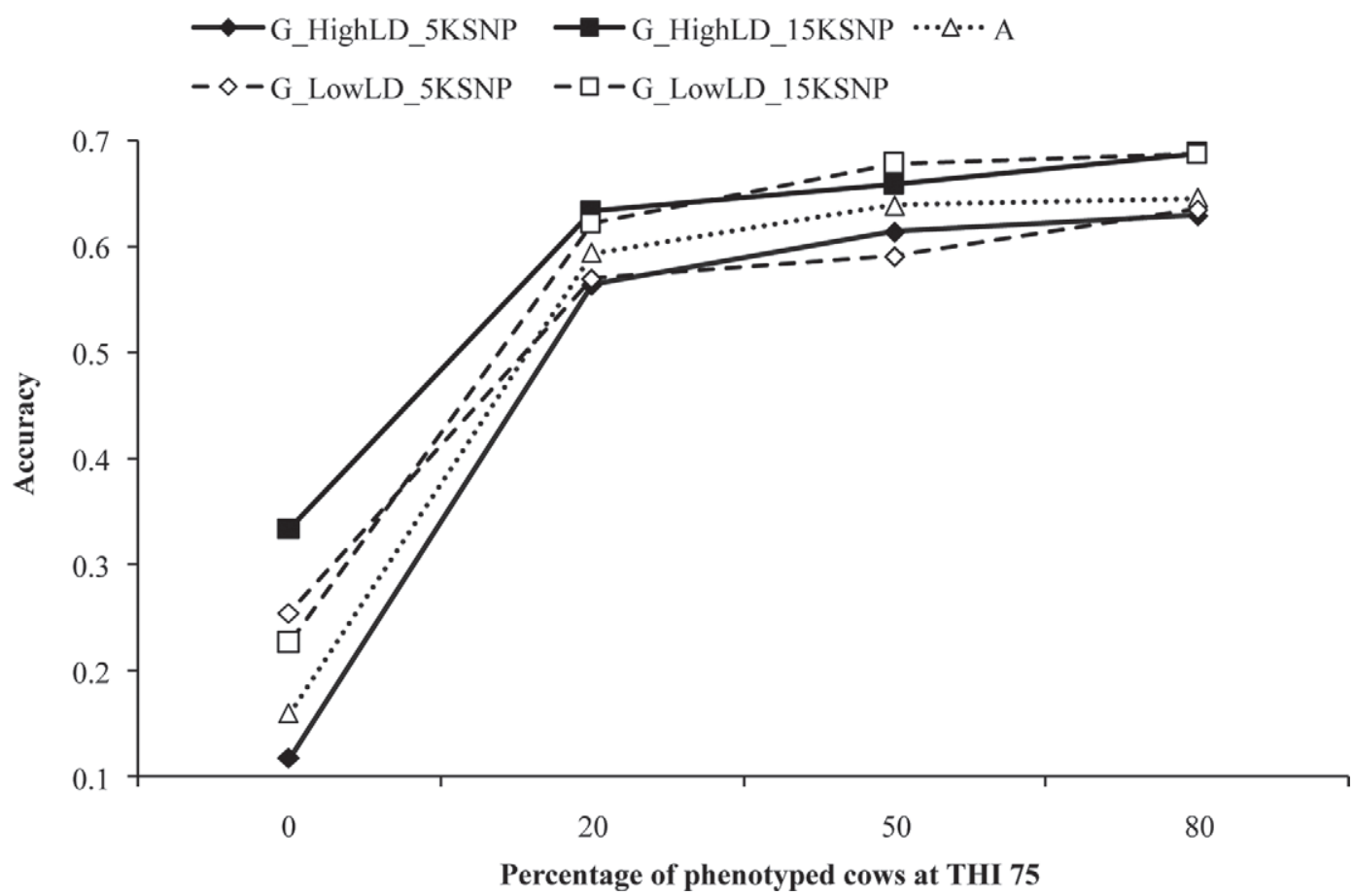

Figure 5. Average accuracies of genomic predictions for non-phenotyped cows at a temperature-humidity index (THI) of 75 for $0,20,50$, and $80 \%$ of cows with phenotypic records at THI 75 with heritabilities of $0.30,0.35,0.40,0.40$, and 0.35 for THI 15, 30, 45, 60 , and 75 , respectively. Different scenarios include variation in SNP density [5,000 (5K) and 15,000 (15K)] and linkage disequilibrium (LD). G = scenario based on the genetic relationship matrix using the SNP data of cows; $\mathrm{A}=$ scenario based on the pedigree relationship matrix.

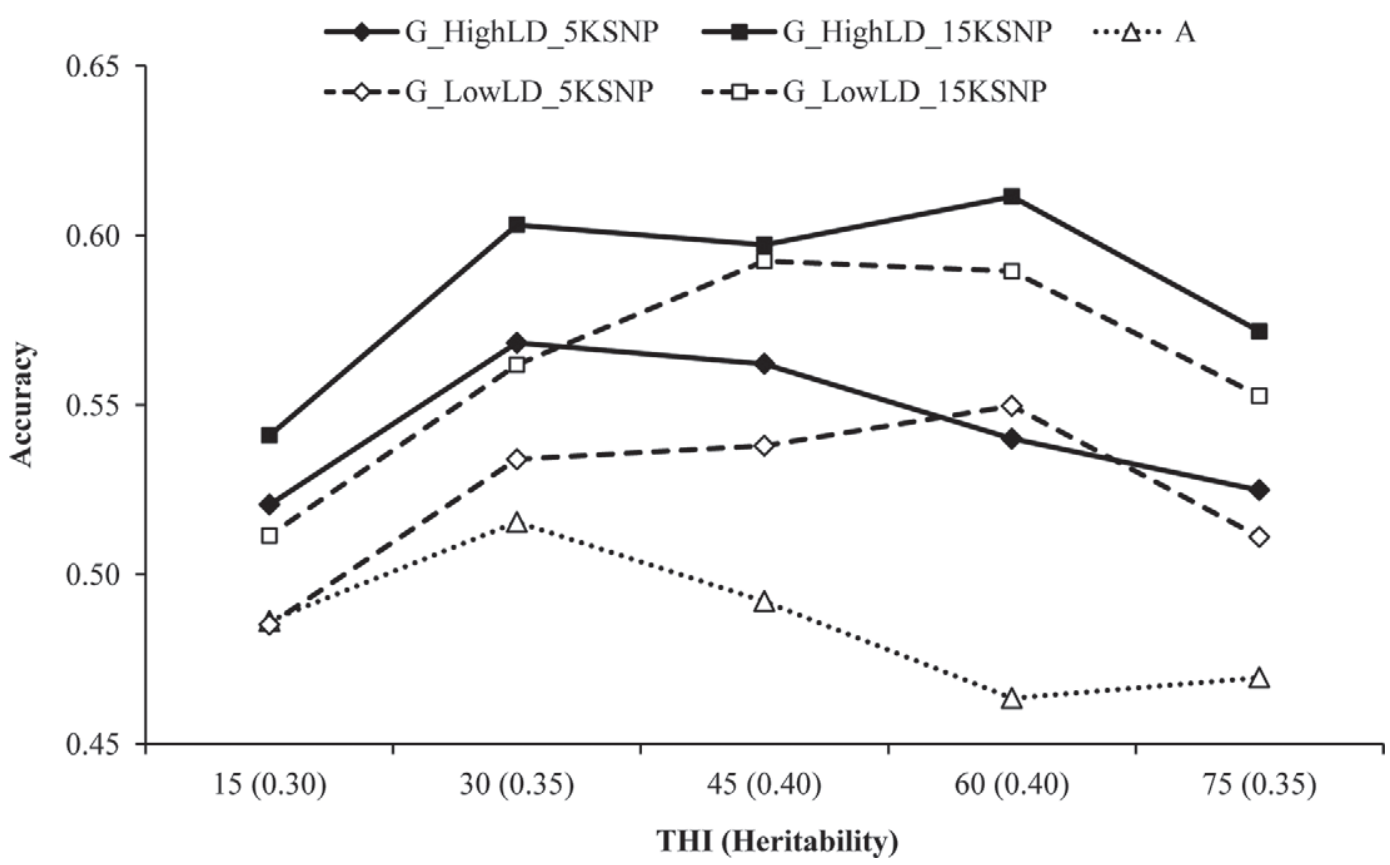

Figure 6. Average accuracies of genomic predictions for the scenarios with 400 cows in the prediction set and 1,600 cows in the calibration set with heritabilities of $0.30,0.35,0.40,0.40$, and 0.35 for temperature-humidity indices (THI) of $15,30,45$, 60 , and 75 , respectively. Different scenarios include variation in SNP density $[5,000(5 \mathrm{~K})$ and $15,000(15 \mathrm{~K})]$ and linkage disequilibrium $(\mathrm{LD})$. G $=$ scenario based on the genetic relationship matrix using the SNP data of cows; A = scenario based on the pedigree relationship matrix. 
Table 5. Average pedigree- and genomic-based relationships for cows within the calibration and the prediction population, and between the 2 populations ${ }^{1}$

\begin{tabular}{lccccrr}
\hline & \multicolumn{5}{c}{ Relationship } \\
\cline { 2 - 6 } Scenario $^{2}$ & Calibration $^{3}$ & Calibration $^{4}$ & Prediction $^{3}$ & Prediction $^{4}$ & Between $^{3}$ & Between $^{4}$ \\
\hline G_LowLD_5KSNP & 0.0008 & 0.0022 & 0.0045 & 0.0285 & -0.0005 & -0.0064 \\
G_LowLD_15KSNP & 0.0008 & 0.0023 & 0.0045 & 0.0291 & -0.0005 & -0.0067 \\
A & 0.0348 & 0.0362 & 0.0382 & 0.0616 & 0.0335 & 0.0277 \\
G_HighLD_5KSNP & 0.0008 & 0.0022 & 0.0044 & 0.0283 & -0.0005 & -0.0064 \\
G_HighLD_15KSNP & 0.0008 & 0.0022 & 0.0044 & 0.0287 & -0.0005 & -0.0068 \\
\hline
\end{tabular}

${ }^{1}$ Averages are from 10 replicates.

${ }^{2} \mathrm{G}=$ scenario based on the genetic relationship matrix using the SNP data of cows; $\mathrm{A}=$ scenario based on the pedigree relationship matrix; $\mathrm{LD}=$ linkage disequilibrium; $5 \mathrm{~K}=5,000 ; 15 \mathrm{~K}=15,000$.

${ }^{3}$ Four hundred cows were randomly allocated to the prediction population; the remaining cows $(1,600)$ were assigned to the calibration population.

${ }^{4}$ Daughters of 8 sires were allocated to the prediction population; the remaining cows were assigned to the calibration population.

sires. The cows from the remaining 32 sires comprised the training set. Hence, the average pedigree-based genetic relationships between cows in the calibration sets and cows in the prediction sets were lower, and ranged from 3.35 to $2.77 \%$ (Table 5 ). The accuracy of predictions for the 5 scenarios decreased, on average, by $25 \%$. In detail, the accuracy was 0.34 for scenarios with pedigree information, 0.44 for G_HighLD_15KSNP, and 0.44 for G_LowLD_15KSNP. Corresponding accuracies were $0.49,0.59$, and 0.56 when 400 cows were randomly allocated to the prediction set. Also for gRRM, the results indicate that genetic relationships between cows in the calibration set and cows in the prediction set played an important role with regard to realized accuracies of predictions. However, accuracies that result from genetic relationships will erode in the long term, and only the effects of SNP in LD with QTL are persistent across populations and generations (Hayes et al., 2009).

Moderately accurate genomic predictions with gRRM also for cows that are completely non-phenotyped (i.e., without phenotypes across THI) allow further improvements with regard to on-farm selection strategies. The availability of a cheap low-density SNP chip implies an extension of genotyping from preselected bull dams toward cohorts of heifers or young cows in commercial dairy cattle herds (e.g., Achler, 2013). Additionally, efficient imputing strategies for totally ungenotyped animals that use information from genotyped relatives are developed for practical application (Pimentel et al., 2013). Application of those imputing techniques will enlarge the pool of genotyped animals while keeping costs constant. Hence, the lack of phenotypes is the most limiting factor for novel traits that are difficult or expensive to measure (e.g., traits related to health, product quality, or animal welfare). Hence, reliable GBV can be predicted when genotyping cows on a large scale, but only phenotyping a subset of the genotyped cows for novel traits. Such a scenario might be relevant, for example, for longitudinal health traits as recorded in contract herds (Gernand et al., 2012).

\section{CONCLUSIONS}

In the present study, first we developed a strategy for simulating longitudinal phenotypic records along with marker information. This strategy is based on differentiated gene or QTL expression on a continuous environmental scale and might be helpful for future evaluations of breeding strategies based on longitudinal data sets. From a methodological point of view, results from this study revealed higher accuracies of predictions when replacing the traditional pedigree-based genetic relationship matrix with the realized genomic relationship matrix in RRM applications. The simultaneous use of genetically correlated longitudinal data in gRRM can predict genetic values of animals without phenotypes. Only a small proportion of cows (i.e., 20\%) with phenotypes at the extreme ends of an environmental scale (here, THI 75) is required to predict GBV of non-phenotyped cows at THI 75. For this scenario, accuracies of predictions of GBV from gRRM for completely non-phenotyped cows were of moderate size and ranged between 0.50 and 0.60 . For a minimized genetic relationship between the calibration and the prediction population, accuracies of genomic predictions decreased for various scenarios (scenarios included variations in LD and of SNP density) by 20 to $30 \%$.

\section{ACKNOWLEDGMENTS}

This study was supported by the Ministry for Science and Culture of Lower Saxony (Hanover, Germany) within the network "KLIFF" - climate impact and adaptation research in Lower Saxony. 


\section{REFERENCES}

Achler, E. 2013. Evaluation of intra-herd selection strategies for genotyped females: Genetic gain, accuracy and economic parameters. MS Thesis. University of Göttingen, Göttingen, Germany.

Aguilar, I., I. Misztal, and S. Tsuruta. 2009. Genetics components of heat stress for dairy cattle with multiple lactations. J. Dairy Sci. 92:5702-5711

Bohmanova, J., I. Misztal, S. Tsuruta, H. D. Norman, and T. J. Lawlor. 2005. National genetic evaluation of milk yield for heat tolerance of United States Holsteins. Interbull Bull. 33:160-162.

Bolormaa, S., J. E. Pryce, B. J. Hayes, and M. E. Goddard. 2010. Multivariate analysis of a genome-wide association study in dairy cattle. J. Dairy Sci. 93:3818-3833.

Brügemann, K., E. Gernand, U. U. von Borstel, and S. König. 2011. Genetic analyses of protein yield in dairy cows applying random regression models with time-dependent and temperature $\times$ humidity-dependent covariates. J. Dairy Sci. 94:4129-4139.

Brügemann, K., E. Gernand, U. König von Borstel, and S. König. 2012. Defining and evaluating heat stress thresholds in different dairy cow production systems. Arch. Tierzucht 55:13-24.

Buch, L. H., M. Kargo, P. Berg, J. Lassen, and A. C. Sørensen. 2012. The value of cows in reference populations for genomic selection of new functional traits. Animal 6:880-886.

Cammack, K. M., E. Antoniou, L. Hearne, and W. R. Lamberson 2009. Testicular gene expression in male mice divergent for fertility after heat stress. Theriogenology 71:651-661.

Cammack, K. M., H. Mesa, and W. R. Lamberson. 2006. Genetic variation in fertility of heat-stressed male mice. Theriogenology 66:2195-2201

Daetwyler, H. D., R. Pong-Wong, B. Villanueva, and J. A. Woolliams. 2010. The impact of genetic architecture on genome-wide evaluation methods. Genetics 185:1021-1031.

DHV (Deutscher Holstein Verband). 2013. German Holsteins 2012 Facts \& Figures. Page 2. Accessed Apr. 10, 2013. http://www. holstein-dhv.de/services/files/statistik/DHV_Einlegeblatt_ZA_ EN.pdf.

Gernand, E., P. Rehbein, U. U. von Borstel, and S. König. 2012. Incidences of and genetic parameters for mastitis, claw disorders, and common health traits recorded in dairy cattle contract herds. J. Dairy Sci. 95:2144-2156.

Goddard, M. 2009. Genomic selection: Prediction of accuracy and maximisation of long term response. Genetica 136:245-257.

Hayes, B. J., P. J. Bowman, A. J. Chamberlain, and M. E. Goddard 2009. Invited review: Genomic selection in dairy cattle: Progress and challenges. J. Dairy Sci. 92:433-443.

Henderson, C. R. 1984. Applications of Linear Models in Animal Breeding. 3rd ed. L. R. Schaeffer, ed. University of Guelph, Guelph, ON, Canada.

Hill, W. G., and A. Robertson. 1968. Linkage disequilibrium in finite populations. Theor. Appl. Genet. 38:226-231.

Jiménez-Montero, J. A., O. González-Recio, and R. Alenda. 2013. Comparison of methods for the implementation of genome-assisted evaluation of Spanish dairy cattle. J. Dairy Sci. 96:625-634.

Kirkpatrick, M., D. Lofsvold, and M. Bulmer. 1990. Analysis of the inheritance, selection and evolution of growth trajectories. Genetics 124:979-993.

Madsen, P., and J. Jensen. 2010. An user's guide to DMU: A package for analysing multivariate mixed models. Version 6 , release 5.0 University of Aarhus, Tjele, Denmark.
Meuwissen, T. H. E. 2009. Accuracy of breeding values of 'unrelated individuals predicted by dense SNP genotyping. Genet. Sel. Evol. 41:35.

Misztal, I., I. Aguilar, S. Tsuruta, J. P. Sanchez, and B. Zumbach 2010. Studies on heat stress in dairy cattle and pigs. Abstract no. 0625 in Proc. of the 9th World Congr. Genet. Appl. Livest. Prod., Leipzig, Germany. German Society for Animal Science, Leipzig, Germany.

Pimentel, E. C. G., M. Wensch-Dorendorf, S. König, and H. H. Swalve. 2013. Enlarging a training set for genomic selection by imputation of un-genotyped animals in populations of varying genetic architecture. Genet. Sel. Evol. 45:12.

Pintus, M. A., E. L. Nicolazzi, J. B. C. H. M. Van Kaam, S. Biffani, A. Stella, G. Gaspa, C. Dimauro, and N. P. P. Macciotta. 2013 Use of different statistical models to predict direct genomic values for productive and functional traits in Italian Holsteins. J. Anim. Breed. Genet. 130:32-40.

Ravagnolo, O., and I. Misztal. 2000. Genetic component of heat stress in dairy cattle, parameter estimation. J. Dairy Sci. 83:2126-2130.

Sargolzaei, M., and F. S. Schenkel. 2009. QMSim: A large-scale genome simulator for livestock. Bioinformatics 25:680-681.

Schaeffer, L. R. 2004. Application of random regression models in animal breeding. Livest. Prod. Sci. 86:35-45.

Schaeffer, L. R., and J. C. M. Dekkers. 1994. Random regressions in animal models for test-day production in dairy cattle. Pages 443-446 in Proc. 5th World Congr. Genet. Appl. Livest. Prod. Guelph, ON, Canada. University of Guelph, Guelph, ON, Canada.

Schierenbeck, S., F. Reinhardt, R. Reents, H. Simianer, and S. König. 2011. Identification of informative cooperator herds for progeny testing based on yield deviations. J. Dairy Sci. 94:2071-2082.

Su, G.-S., and P. Madsen. 2011. User's guide for Gmatrix: A program for computing genomic relationship matrix. University of Aarhus, Tjele, Denmark.

Suchocki, T., and J. Szyda. 2011. Statistical modelling of growth using a mixed model with orthogonal polynomials. J. Appl. Genet. 52:95-100.

Tsuruta, S., I. Misztal, I. Aguilar, and T. J. Lawlor. 2011. Multipletrait genomic evaluation of linear type traits using genomic and phenotypic data in US Holsteins. J. Dairy Sci. 94:4198-4204.

VanRaden, P. M. 2008. Efficient methods to compute genomic predictions. J. Dairy Sci. 91:4414-4423.

Verbyla, K. L., M. P. L. Calus, H. A. Mulder, Y. de Haas, and R. F. Veerkamp. 2010. Predicting energy balance for dairy cows using high-density single nucleotide polymorphism information. J. Dairy Sci. 93:2757-2764.

Weigel, K. A., P. C. Hoffman, W. Herring, and T. J. Lawlor Jr. 2012 Potential gains in lifetime net merit from genomic testing of cows, heifers, and calves on commercial dairy farms. J. Dairy Sci 95:2215-2225

Yin, T., B. Bapst, U. U. v. Borstel, H. Simianer, and S. König. 2012 Genetic parameters for Gaussian and categorical in organic and low input dairy herds based on random regression methodology. Livest. Sci. 147:159-169.

Zhong. S., J. C. M. Dekkers, R. L. Fernando, and J.-L. Jannink. 2009 Factors affecting accuracy from genomic selection in populations derived from multiple inbred lines: A barley case study. Genetics $182: 355-364$. 